\title{
Energetic particle fluxes in the exterior cusp and the high-latitude dayside magnetosphere: statistical results from the Cluster/RAPID instrument
}

\author{
T. Asikainen and K. Mursula \\ Department of Physical Sciences, University of Oulu, Finland
}

Received: 25 February 2004 - Revised: 2 March 2005 - Accepted: 24 May 2005 - Published: 15 September 2005

\begin{abstract}
In this paper we study the fluxes of energetic protons $(30-4000 \mathrm{keV})$ and electrons $(20-400 \mathrm{keV})$ in the exterior cusp and in the adjacent high-latitude dayside plasma sheet (HLPS) with the Cluster/RAPID instrument. Using two sample orbits we demonstrate that the Cluster observations at high latitudes can be dramatically different because the satellite orbit traverses different plasma regions for different external conditions. We make a statistical study of energetic particles in the exterior cusp and HLPS by analysing all outbound Cluster dayside passes in February and March, 2002 and 2003. The average particle fluxes in HLPS are roughly three (protons) or ten (electrons) times larger than in the exterior cusp. This is also true on those Cluster orbits where both regions are visited within a short time interval. Moreover, the total electron fluxes, as well as proton fluxes above some $100 \mathrm{keV}$, in these two regions correlate with each other. This is true even for fluxes in every energy channel when considered separately. The spectral indices of electron and proton fluxes are the same in the two regions. We also examine the possible dependence of particle fluxes at different energies on the external (solar wind and IMF) and internal (geomagnetic) conditions. The energetic proton fluxes (but not electron fluxes) in the cusp behave differently at low and high energies. At low energies $(<70 \mathrm{keV})$, the fluxes increase strongly with the magnitude of IMF $B_{y}$. Instead, at higher energies the proton fluxes in the cusp depend on substorm/geomagnetic activity. In HLPS proton fluxes, irrespective of energy, depend strongly on the $K_{p}$ and AE indices. The electron fluxes in HLPS depend both on the $K_{p}$ index and the solar wind speed. In the cusp the electron fluxes mainly depend on the solar wind speed, and are higher for northward than southward IMF. These results give strong evidence in favour of the idea that the high-latitude dayside plasma sheet is the main source of energetic particles in the exterior cusp. Energetic particles can reach HLPS from the near-Earth tail. The closed field lines of HLPS act
\end{abstract}

Correspondence to: T. Asikainen

(timo.asikainen@oulu.fi) as storage for these particles. Direct diffusion (for electrons and high-energy protons) and magnetic reconnection in the high-latitude magnetopause near HLPS (for low energy protons) control the number of particles released into the exterior cusp. Note that this explanation, in contrast to other suggested theories, works both for the energetic protons and electrons in the exterior cusp.

Keywords. Magnetospheric physics (energetic particles, trapped; magnetopause, cusp and boundary layers; solar wind-magnetosphere interactions)

\section{Introduction}

According to the classical picture of the magnetosphere, the high-latitude dayside magnetosphere and the adjacent cusp regions cannot trap particles stably (Roederer, 1970). Still, a number of recent studies (Fritz et al., 1999; Chen et al., 2001; Fritz, 2001; Fritz et al., 2003) have reported high fluxes of energetic particles in the exterior cusp. Detailed theoretical studies based on realistic magnetic field models have also revealed that the high-latitude dayside regions can quasi-stably trap energetic particles whose motion is primarily governed by the magnetic gradient-curvature drift rather than the electric drift (Sheldon et al., 1998). Accordingly, there is considerable observational evidence and some theoretical support for a significant flux of energetic particles in the cusp. However, the origin of these particle populations in the cusp is less clear. Chen et al. (1998) have suggested that the energetic particles in the exterior cusp are accelerated locally by electromagnetic waves. Chang et al. (1998) and Trattner et al. (2001) have argued that the lower energy particles may be accelerated at the quasi-parallel bow shock, entering from there into the exterior cusp along field lines connecting the two regions, while the very high energy particles may diffuse to the cusp from the inner magnetosphere. Kremser et al. (1995) studied the low-altitude cusp and showed that energetic $(E \approx 50 \mathrm{keV})$ particles in the cusp come from the 
magnetosphere and that a separate, low-energy $(<15 \mathrm{keV})$ ion population in the cusp is of magnetosheath origin.

While energetic particle fluxes in the cusp have received keen interest during the last few years, the corresponding fluxes in the closed field line region of the high-latitude dayside plasma sheet (HLPS) have remained with less consideration. However, before the question of the origin of energetic particles in the cusp can be resolved, it is mandatory to know more about the energetic particle fluxes in the adjacent closed field lines.

In this work we define the exterior cusp classically as the high-altitude part of the cusp funnel (or cusp proper) where magnetosheath plasma has more or less direct access. Because of stagnant high-density plasma the exterior cusp is essentially a diamagnetic cavity where the magnetic field strength is considerably lower than in the surroundings. Momentarily the field strength may even be close to zero. Large fluctuations in the field direction and strength occur frequently. On the other hand, similar large diamagnetic cavities are not seen so clearly in the cusp funnel. The highlatitude dayside plasma sheet is a region of closed field lines equatorward of the cusp, where the magnetic field is stable and thermal plasma density is low.

In this paper we study the energetic proton and electron fluxes in both the exterior cusp and HLPS using the Cluster RAPID instrument (Wilken et al., 1997) which measures protons and electrons in the energy range of about $30-4000 \mathrm{keV}$ and $20-400 \mathrm{keV}$, respectively. We also examine the dependence of these fluxes on the external (solar wind plasma and IMF) and internal (geomagnetic, ring current) conditions, in order to clarify the origin of energetic particles in the cusp region, and the related question of how these particles are accelerated and transported into this region.

\section{Data and method}

The orbit of the Cluster-II satellites is well suited for studying the dayside high-latitude regions. Around vernal equinoxes the orbit apogee is at the dayside and the satellites typically fly from the nightside over the polar cap into the dayside, passing through the high-latitude boundary layer poleward of the cusp. In the dayside the satellites typically enter either the exterior cusp or (perhaps after a brief visit to the cusp funnel) the closed field lines of the high-latitude dayside plasma sheet, depending on the orientation of the magnetosphere and the external conditions. Finally, after the exterior cusp or HLPS, the satellites continue to the magnetosheath and through the bow shock to the solar wind.

The exterior cusp was defined as a region of turbulent and depressed magnetic field with a high plasma density (Chen et al., 1998) and the HLPS as a region of closed field lines where thermal plasma density is low and the magnetic field is fairly steady. Accordingly, we have identified these regions mainly on the basis of their different properties in magnetic field and plasma density. For plasma density we mostly used the data obtained from the CIS instrument (Rème et al.,
1997) or the spacecraft potential data from the EFW instrument (Gustafsson et al., 1997) whenever CIS data was not available. Magnetic field data was measured by the FGM instrument (Balogh et al., 1997).

In the statistical analysis we study the fluxes of energetic particles in the exterior cusp and HLPS for different external and internal conditions. The measurements of solar wind and the interplanetary magnetic field were obtained from the ACE (Advanced Composition Explorer) spacecraft (Stone et al., 1998). Since ACE is far upstream the measurements do not coincide with the timing of magnetospheric phenomena and a temporal correction is necessary. Since IMF is frozen into the solar wind stream the magnetic field propagates toward the Earth at the momentary solar wind speed. If the distance from ACE to the Earth along the Sun-Earth line is denoted by $X$, the momentary time delay from ACE to Earth is

$\Delta T(t)=X / V(t)$,

where $V(t)$ is the momentary solar wind speed. Time shifted IMF and solar wind measurements are obtained by the following transformation

$B(t) \rightarrow B(t+\Delta T(t))$

i.e. a variable time delay is added to each measured data point. Using the 1-min time resolution for solar wind measurements, the temporal changes in solar wind speed were fairly slow, so that the time order of measurements at ACE and the Earth mostly remained the same. The average difference in the time delay calculated by the variable solar wind speed compared to that obtained from a constant speed was about $2 \mathrm{~min}$.

\section{Sample Cluster orbits}

Figure 1 (left-hand side) shows a sample plot of Cluster data from 2-3 February 2003. At the beginning of the 8-h time interval the magnetic field is quite smooth, both in direction and intensity, and the plasma density is very low. At this time the satellite flies in the open field lines of the northern tail lobe. From about 21:20 UT to 22:00 UT the plasma density increases considerably while the magnetic field is still quite smooth, indicating that the satellites are still inside the magnetopause. The region of increasing plasma density consists of a few plasma cells convecting tailwards. Similar convecting plasma cells are a frequent phenomenon observed by Cluster satellites at the dayside, and form the high-latitude boundary layer (HLBL, or plasma mantle) plasma region (Rosenbauer et al., 1975). The increasing density of plasma cells reflects the fact that the satellites are approaching the exterior cusp region where they enter at about 22:00 UT. The cusp is seen here as a region of very turbulent and depressed magnetic field and high plasma density which is comparable to the magnetosheath level. This is a classic signature of a cusp diamagnetic cavity (Chen et al., 1998). Figure 1 shows 

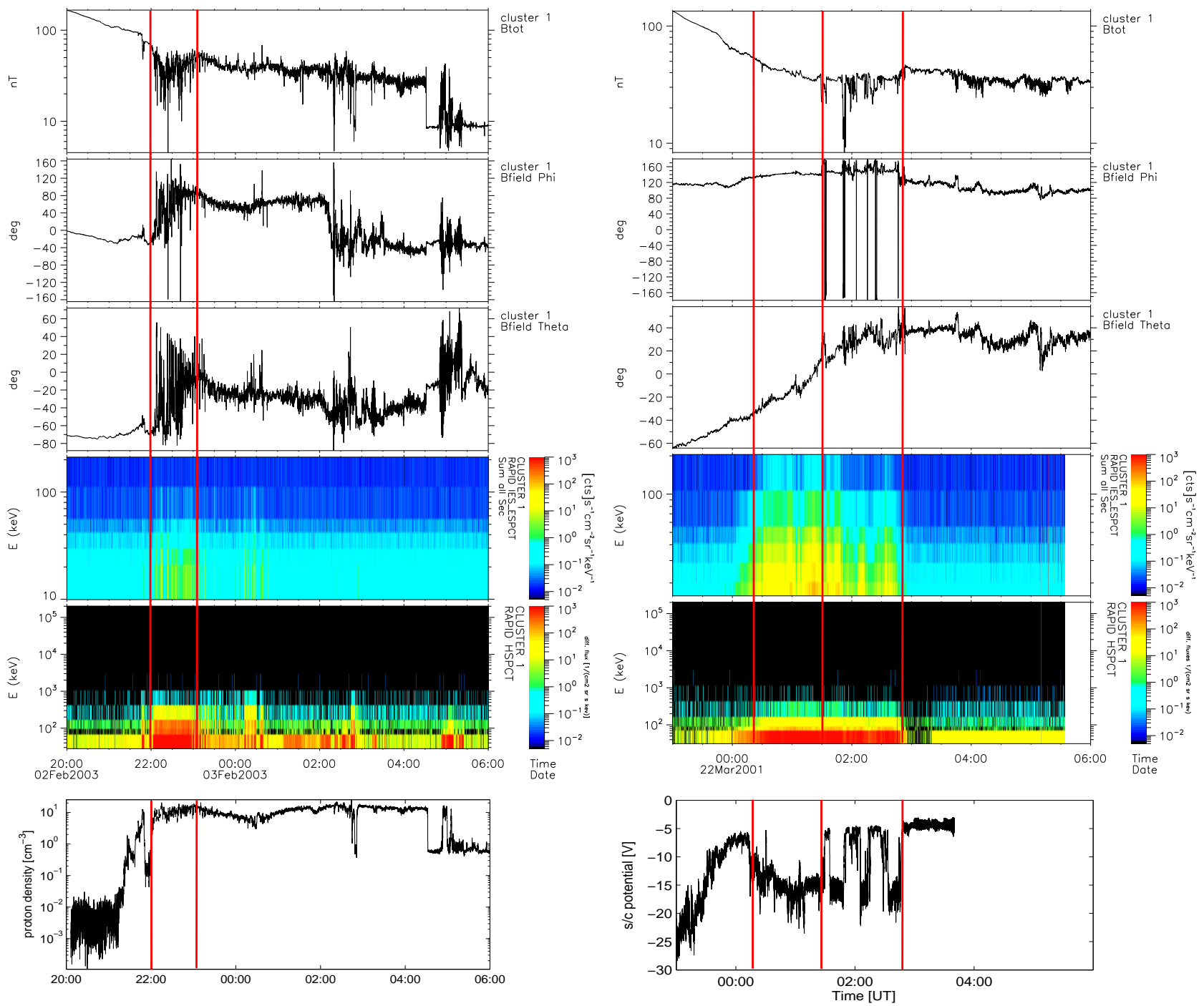

Fig. 1. Sample of Cluster FGM and RAPID data. From top to bottom: total magnetic field intensity, azimuthal angle $(\phi)$ and polar angle $(\theta)$ of magnetic field, electron and proton energy spectra, and plasma density (left side figure) and spacecraft potential (right side figure). Left side: 2 February 2003, right side: 22 March 2001.

that in this case there are significant fluxes of energetic particles present in the exterior cusp.

The satellite leaves the exterior cusp for the magnetosheath at about 23:05 UT. At this time the satellite clearly exits the diamagnetic cavity of the cusp which is seen as an increase in the magnetic field. Furthermore, when comparing the magnetic field observed by Cluster after 23:05 UT with the propagated IMF from ACE (not shown), the two fields resemble each other well, while in the exterior cusp the field deviates noticeably from the propagated and shocked IMF. Also, after 23:05 UT the plasma flow becomes more regular (as indicated by the CIS instrument, not shown). We note that on this pass the Cluster satellites fly from the open fieldline region via the mantle into the exterior cusp, and do not enter at all the closed field line region of the dayside plasma sheet. This is most likely due to the negative IMF $B_{z}$ component during this time. The satellites remain mainly within the magnetosheath until about 04:30 UT, except for a number of earlier bow shock crossings like the one at about 02:50 UT. Figure 1 depicts that there are a number of energetic particles, mostly energetic protons, even in the magnetosheath. (There are also heavy ions in the magnetosheath (not shown) that are even more widely spread than protons, mostly likely due to their larger gyroradii.) However, the maximum energetic particle fluxes are seen in the exterior cusp. Proton (and heavy ion) fluxes decrease towards the bow shock, suggesting that the bow shock is not a significant source of those energetic particles that were observed in the exterior cusp. Note also that the sharp bow shock crossing at 04:30 UT is not related to an increase in energetic particle fluxes. Thus, the moving bow shock does not produce a significant amount of energetic particles in the magnetosheath.

Figure 1 (right-hand side) shows the Cluster orbit on 22 March 2001, which has been discussed in more detail 
Table 1. A list of the times when Cluster 4 observed the exterior cusp.

\begin{tabular}{llll}
\hline Date & $\begin{array}{l}\text { Entering } \\
\text { time }\end{array}$ & $\begin{array}{l}\text { Exit } \\
\text { time }\end{array}$ & $\begin{array}{l}\text { duration } \\
{[\mathrm{min}]}\end{array}$ \\
\hline 01 Feb. 2002 & $12: 00$ & $12: 45$ & 45 \\
11 Feb. 2002 & $00: 00$ & $01: 20$ & 80 \\
20 Feb. 2002 & $12: 55$ & $14: 00$ & 65 \\
22 Feb. 2002 & $22: 30$ & $23: 37$ & 67 \\
04 Mar. 2002 & $08: 50$ & $09: 50$ & 60 \\
21 Mar. 2002 & $00: 45$ & $02: 10$ & 85 \\
02 Feb. 2003 & $21: 40$ & $23: 05$ & 85 \\
07 Feb. 2003 & $17: 45$ & $18: 30$ & 45 \\
12 Feb. 2003 & $11: 05$ & $11: 20$ & 15 \\
14 Feb. 2003 & $18: 40$ & $19: 20$ & 40 \\
14 Feb. 2003 & $19: 50$ & $20: 18$ & 28 \\
26 Feb. 2003 & $15: 43$ & $16: 40$ & 117 \\
03 Mar. 2003 & $10: 48$ & $10: 58$ & 10 \\
03 Mar. 2003 & $11: 30$ & $12: 05$ & 35 \\
05 Mar. 2003 & $19: 40$ & $21: 00$ & 80 \\
15 Mar. 2003 & $07: 30$ & $08: 26$ & 56 \\
17 Mar. 2003 & $17: 20$ & $18: 10$ & 50 \\
17 Mar. 2003 & $18: 57$ & $19: 20$ & 23 \\
22 Mar. 2003 & $11: 00$ & $12: 50$ & 110 \\
\hline
\end{tabular}

by Mursula et al. (2005). During this dayside orbit the IMF $B_{z}$ component is positive and the satellites pass from the nightside through the cusp funnel at about 00:0000:15 UT into the high-latitude dayside plasma sheet at about 00:20 UT. Significant fluxes of energetic particles (electrons, protons and heavy ions) were observed throughout the region until a final crossing into the magnetosheath at about 02:50 UT. While mainly inside the magnetosphere, the satellites crossed the high-latitude dayside magnetopause to the magnetosheath several times between 01:30 UT and 02:50 UT, which could be seen as multiple increases of magnetosheath plasma in conjuction with simultaneous dropouts in magnetic field intensity and energetic particle fluxes. By comparing this orbit to the one discussed above, we can see that the Cluster satellites fly in very different plasma regions, depending on the direction of the IMF $\left(B_{z}\right.$ was about $-4 \mathrm{nT}$ on 2 February 2003 and $+6 \mathrm{nT}$ on 22 March 2001) and the dipole tilt angle (the dipole tilt was about $-14^{\circ}$ on 2 February 2003 and about $-5^{\circ}$ on 22 March 2001). These factors (among others like solar wind pressure) profoundly affect whether the satellites mainly fly at the dayside in the closed field lines of the high-latitude plasma sheet or in the exterior cusp region. Also, the energetic particle observations are quite different in these two cases. Accordingly, when studying energetic particle fluxes at the dayside, it is mandatory to know which regions the satellites are actually sampling.

\section{Statistical analysis}

For our statistical analysis we selected a total of 29 outbound Cluster orbits crossing the dayside regions in the Northern
Table 2. A list of the times when Cluster 4 observed the highlatitude dayside magnetosphere.

\begin{tabular}{llll}
\hline Date & $\begin{array}{l}\text { Entering } \\
\text { time }\end{array}$ & $\begin{array}{l}\text { Exit } \\
\text { time }\end{array}$ & $\begin{array}{l}\text { duration } \\
\text { [min] }\end{array}$ \\
\hline 15 Feb. 2002 & $21: 35$ & $23: 40$ & 125 \\
18 Feb. 2002 & $03: 30$ & $05: 00$ & 90 \\
02 Mar. 2002 & $02: 20$ & $03: 30$ & 70 \\
09 Mar. 2002 & $04: 00$ & $06: 25$ & 145 \\
05 Feb. 2003 & $06: 30$ & $11: 10$ & 280 \\
10 Feb. 2003 & $03: 00$ & $04: 15$ & 75 \\
12 Feb. 2003 & $09: 30$ & $11: 05$ & 95 \\
17 Feb. 2003 & $03: 25$ & $04: 20$ & 55 \\
17 Feb. 2003 & $04: 24$ & $07: 15$ & 171 \\
21-22 Feb. 2003 & $22: 30$ & $01: 05$ & 215 \\
24 Feb. 2003 & $06: 05$ & $10: 30$ & 265 \\
01 Mar. 2003 & $02: 00$ & $04: 15$ & 135 \\
03 Mar. 2003 & $10: 58$ & $11: 30$ & 32 \\
08 Mar. 2003 & $04: 20$ & $06: 53$ & 153 \\
15 Mar. 2003 & $08: 30$ & $09: 17$ & 238 \\
20 Mar. 2003 & $01: 45$ & $04: 43$ & 178 \\
24 Mar. 2003 & $21: 00$ & $23: 30$ & 150 \\
\hline
\end{tabular}

Hemisphere in February and March, 2002 and 2003. The only selection criterium we used was that the regions where the satellites visited could be clearly identified. We have used data from only one Cluster satellite $(\mathrm{C} 4$, the Tango) in the statistical analysis, in order to avoid problems related to possibly insufficient inter-spacecraft calibration of the RAPID instrument. Each dayside pass was analysed in detail and the various plasma regions, in particular the exterior cusp and the dayside high-latitude plasma sheet, were identified on the basis of plasma density and magnetic field properties in these regions (see Introduction). Here one event means a time interval when the satellite was in one of the two above regions. Out of the 29 orbits the exterior cusp was observed in 16 orbits, in some cases more than once, thus adding up to 19 observations of exterior cusp altogether. Similarly, the HLPS was observed in 16 orbits, altogether 17 times. The exterior cusp passes are listed in Table 1 and the HLPS passes are listed in Table 2.

The entrance and exit times of the satellite during each visit in the exterior cusp and high-latitude dayside magnetosphere were registered. Average integral fluxes of energetic electrons and protons were calculated using RAPID observations during these time intervals. (Heavier ions were left out from this analysis.) Furthermore, we calculated the average differential fluxes in the five lowest energy channels for protons $(30-1100 \mathrm{keV})$ and electrons $(20-172 \mathrm{keV})$. Also, the corresponding average values of the IMF components and solar wind parameters were calculated as averages over the same time intervals after the delay due to momentary solar wind velocity was taken into account (see discussion above). We also calculated the $K_{p}$, and $\mathrm{AE}$ indices. Here the $K_{p}$ index was taken as an average over the time interval when the satellite was in a certain region. However, the AE index 

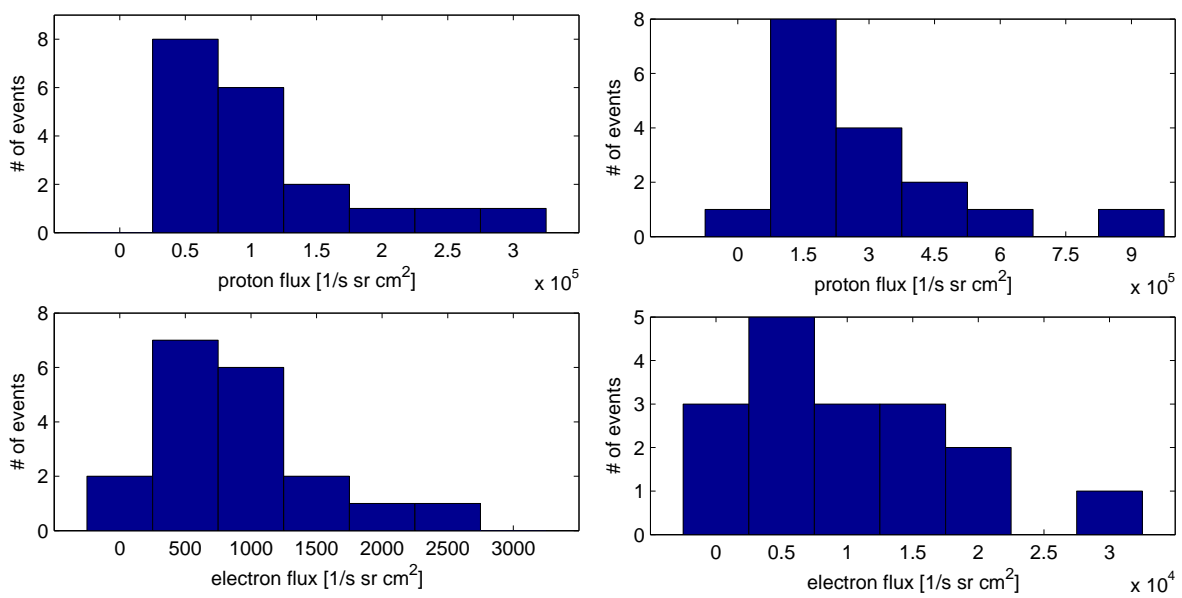

Fig. 2. Histograms of the proton and electron fluxes observed in the exterior cusp (left) and the high-latitude dayside plasma sheet (right).

we used is an average of up to three hours before the satellite entered a region. This three-hour time lag was used in order to take into account the time it takes for a particle to drift from the tail (where substorm acceleration occurs) to the high-latitude dayside regions.

\subsection{Exterior cusp}

Let us first briefly study the position of the exterior cusp region. It is known that the geodipole tilt angle affects the position of the cusp. When the dipole tilt is negative (i.e. the dipole is tilted away from the Sun) the northern cusp is also slightly tilted away from the Sun. Similarly, when the dipole tilt is positive (dipole tilted sunward) the northern cusp is tilted sunward. Using the list of cusp events observed by Cluster, we found a weak but statistically significant correlation between the tilt angle and the IMF $B_{z}$ value. (We use the GSM coordinate system throughout the paper). This correlation implies that certain configurations of the geodipole tilt angle and IMF $B_{z}$ are needed in order for Cluster to meet the exterior cusp. This correlation can naturally be explained in terms of reconnection. When the dipole is strongly tilted away from the Sun and if the IMF did not affect the cusp position, the Cluster satellites would pass from the polar cap through the cusp funnel into the HLPS (and thereafter into magnetosheath), and would reach the exterior cusp less frequently. This roughly corresponds to the orbital situation depicted in the right side of Fig. 1. However, magnetic reconnection taking place at the subsolar point (large $B_{z}<0$ ) can shift the cusp position equatorwards, so that the satellites fly from the polar cap into the exterior cusp and from there into the magnetosheath. This corresponds to the orbital situation depicted in the left side of Fig. 1.

Figure 2 displays the histograms of total proton and electron flux levels in the exterior cusp and high-latitude dayside plasma sheet. The histograms show that in the exterior cusp the average flux of energetic protons is on the order of $10^{5}$ (in units of $\mathrm{cm}^{-2} \mathrm{~s}^{-1} \mathrm{sr}^{-1}$ ) while the electron fluxes are on the order of $10^{3}$, i.e. roughly two orders of magnitude lower.

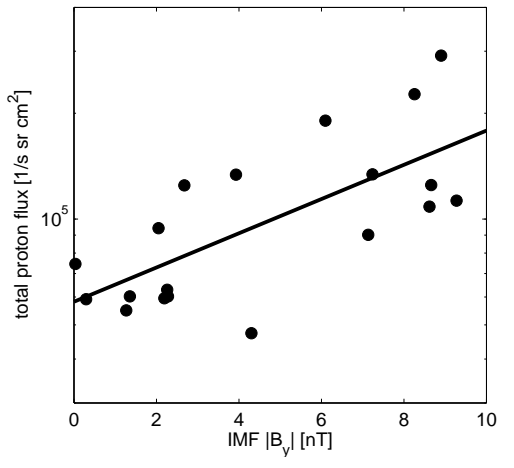

Fig. 3. Total proton fluxes observed in the exterior cusp as a function of IMF $\left|B_{y}\right|$.

In the HLPS the proton fluxes are about 3 times higher and electron fluxes about an order of magnitude higher than in the exterior cusp. Another interesting feature of these histograms is that they closely follow the log-normal distribution. This suggests that the logarithmic scale should be used when analysing the fluxes.

Figure 3 shows the total flux of protons in the exterior cusp as a function of the magnitude of IMF $B_{y}$ component. The figure also displays a least-squares fit to the logarithmic fluxes. Figure 3 shows that the average proton flux in the exterior cusp increases with increasing IMF $B_{y}$ magnitude. The Spearman rank correlation coefficient between the logarithmic total proton flux and the IMF $B_{y}$ magnitude is 0.67 with a P-value of 0.0008 . We have used the nonparametric Spearman rank correlation coefficient instead of the normal (Pearson) correlation because when calculating the P-value as a test of statistical significance, the Spearman method does not require any assumptions about the distribution of the variables while the Pearson method assumes normal distributions. Since neither the fluxes nor the $\left|B_{y}\right|$ values are normally distributed the use of the Pearson correlation would give an erroneous significance estimate for the 

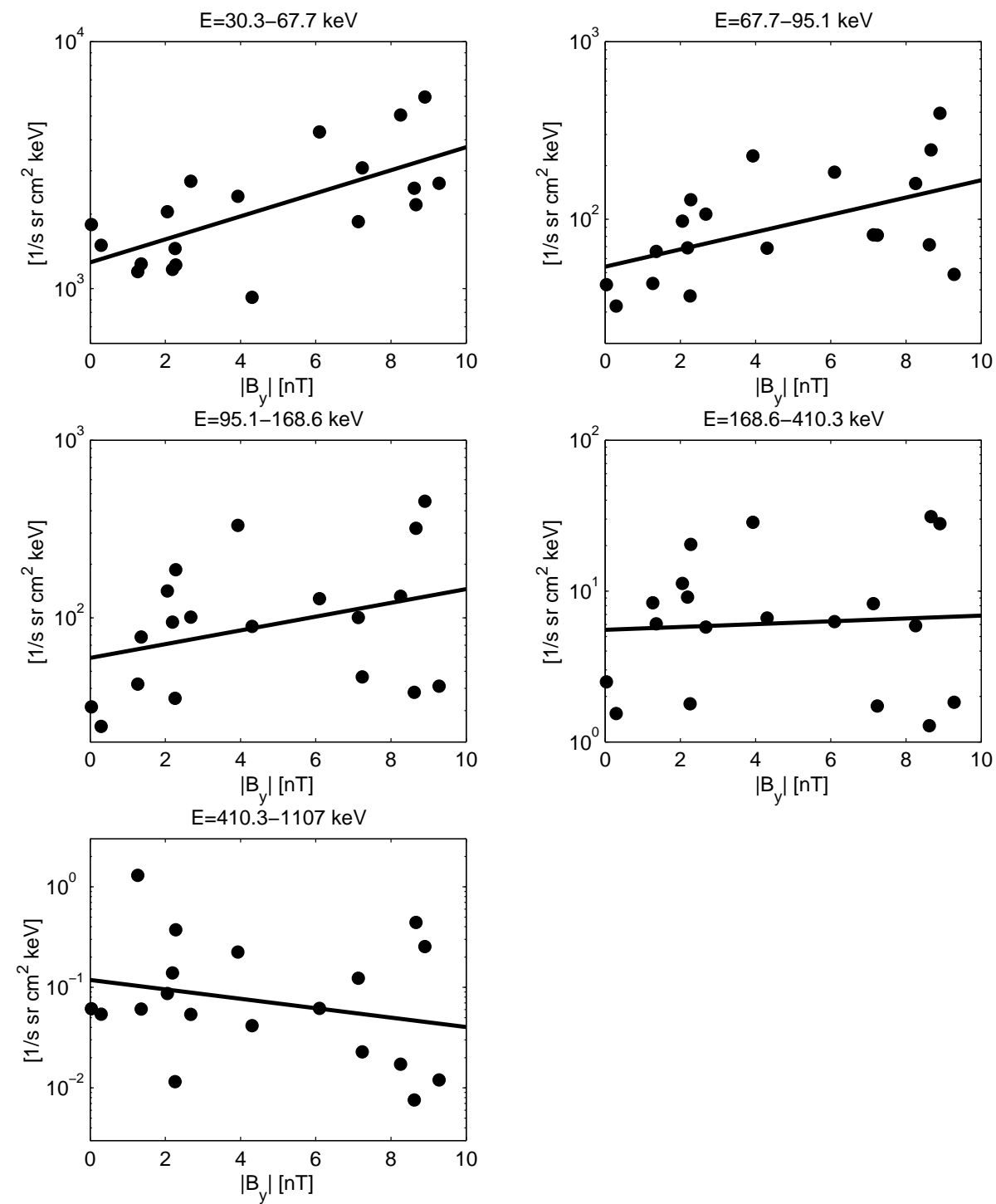

Fig. 4. Differential proton fluxes observed in the exterior cusp at the five lowest energy channels as a function of IMF $\left|B_{y}\right|$.

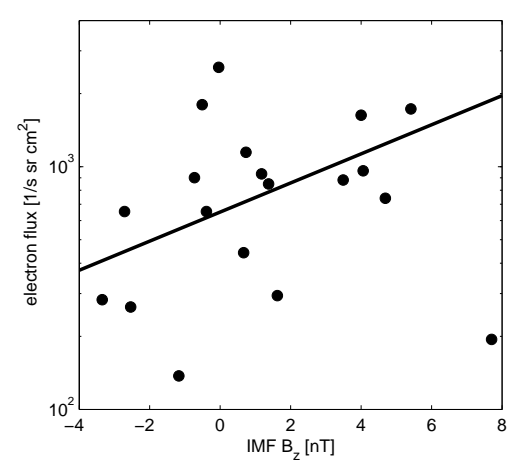

Fig. 5. Total electron fluxes observed in the exterior cusp as a function of IMF $B_{z}$.

correlation coefficient. We have also tested the correlation of the total proton fluxes with IMF $B_{x}$ and $B_{z}$ components, but did not find any significant dependence.
Since particles of different energies often behave differently in the near-Earth magnetosphere, it is interesting to study if there are also differences between protons of different energy in the exterior cusp. We have plotted the differential proton fluxes for the five lowest proton energy channels of the RAPID instrument vs. IMF $B_{y}$ magnitude in Fig. 4. Table 3 depicts the Spearman correlation coefficients and the corresponding P-values, as well as the slopes of the leastsquare fits for each energy channel. Accordingly, it is clear that there is a strong energy dependence hidden in the correlation depicted in Fig. 3 such that the strong correlation exists only at low energies (up to about $100 \mathrm{keV}$ ) whereas no correlation is found at higher energies.

For electron fluxes we found no dependence on the IMF $B_{x}$ and $B_{y}$ components. However, there is a weak dependence on the $B_{z}$ component. This relation is shown in Fig. 5. It seems that the electron fluxes are slightly higher for positive IMF $B_{z}$. Omitting the one outlier at $B_{z} \approx 8 \mathrm{nT}$ 

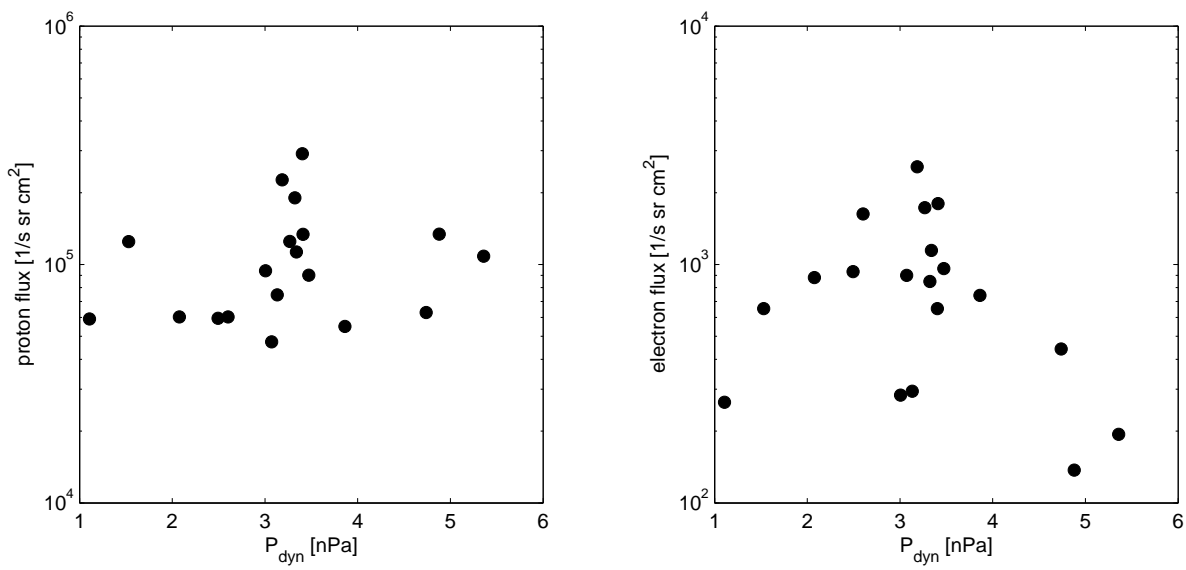

Fig. 6. Total energetic particle fluxes in the exterior cusp as a function of solar wind dynamic pressure. Proton fluxes are shown in the left and electron fluxes in the right.
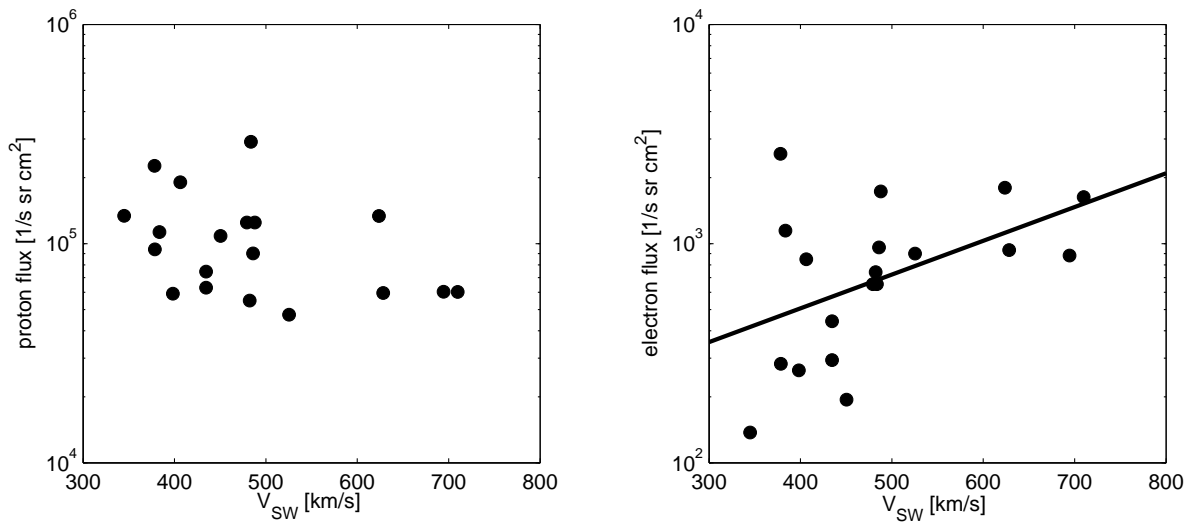

Fig. 7. Total energetic particle fluxes in the exterior cusp as a function of solar wind speed. Proton fluxes are shown in the left and electron fluxes in the right.

the Spearman correlation between the total electron flux and IMF $B_{z}$ is $0.45(\mathrm{P}=0.03)$. The same trend is seen in all energy channels.

Figure 6 shows the proton and electron fluxes in the exterior cusp as a function of solar wind dynamic pressure. There seems to be a clear peak in the electron flux at around $3.2 \mathrm{nPa}$. For proton fluxes there seems to be no clear dependence on solar wind pressure. However, there is a similar but less pronounced peak in the proton flux around $3.2 \mathrm{nPa}$ as for the electrons. In fact, this peak is even more visible in the higher energy channels (not shown). Concerning the pressure we wish to note that the range of pressure values was rather small during the observed passes, reflecting the fairly quiet external conditions during the exterior cusp passes in the four months studied.

Figure 7 displays the total energetic proton and electron fluxes in the exterior cusp as a function of solar wind speed. There is a weak decreasing trend in the proton fluxes with increasing solar wind speed. However, when looking into the different energy channels (see Fig. 8) we see that this decreasing trend only appears in the lowest energy channel.
Table 3. Spearman correlation coefficients between differential proton fluxes in the exterior cusp and magnitude of IMF $B y$ in different energy ranges.

\begin{tabular}{llll}
\hline $\begin{array}{l}\text { Energy range } \\
{[\mathrm{keV}]}\end{array}$ & $\begin{array}{l}\text { Spearman } \\
\text { correlation }\end{array}$ & P-value & $\begin{array}{l}\text { Slope of } \\
\text { fit }\end{array}$ \\
\hline $30.3-67.7$ & 0.66 & 0.001 & 0.1073 \\
$67.7-95.1$ & 0.57 & 0.005 & 0.1122 \\
$95.1-168.6$ & 0.39 & 0.05 & 0.0891 \\
$168.6-410.3$ & 0.07 & 0.38 & 0.0219 \\
$410.3-1107$ & -0.19 & 0.21 & -0.1077 \\
\hline
\end{tabular}

At higher energies the relation is opposite and the fluxes increase with solar wind speed. The Spearman correlation between the proton flux and solar wind speed at the lowest energy channel is $-0.54(\mathrm{P}=0.009)$. On the other hand, the correlation is significantly positive for channels $3-5$. On the fourth energy channel we obtain the highest correlation of 0.59 ( $\mathrm{P}=0.004)$. The right side plot of Fig. 7 shows that the electron fluxes correlate with solar wind speed being higher 

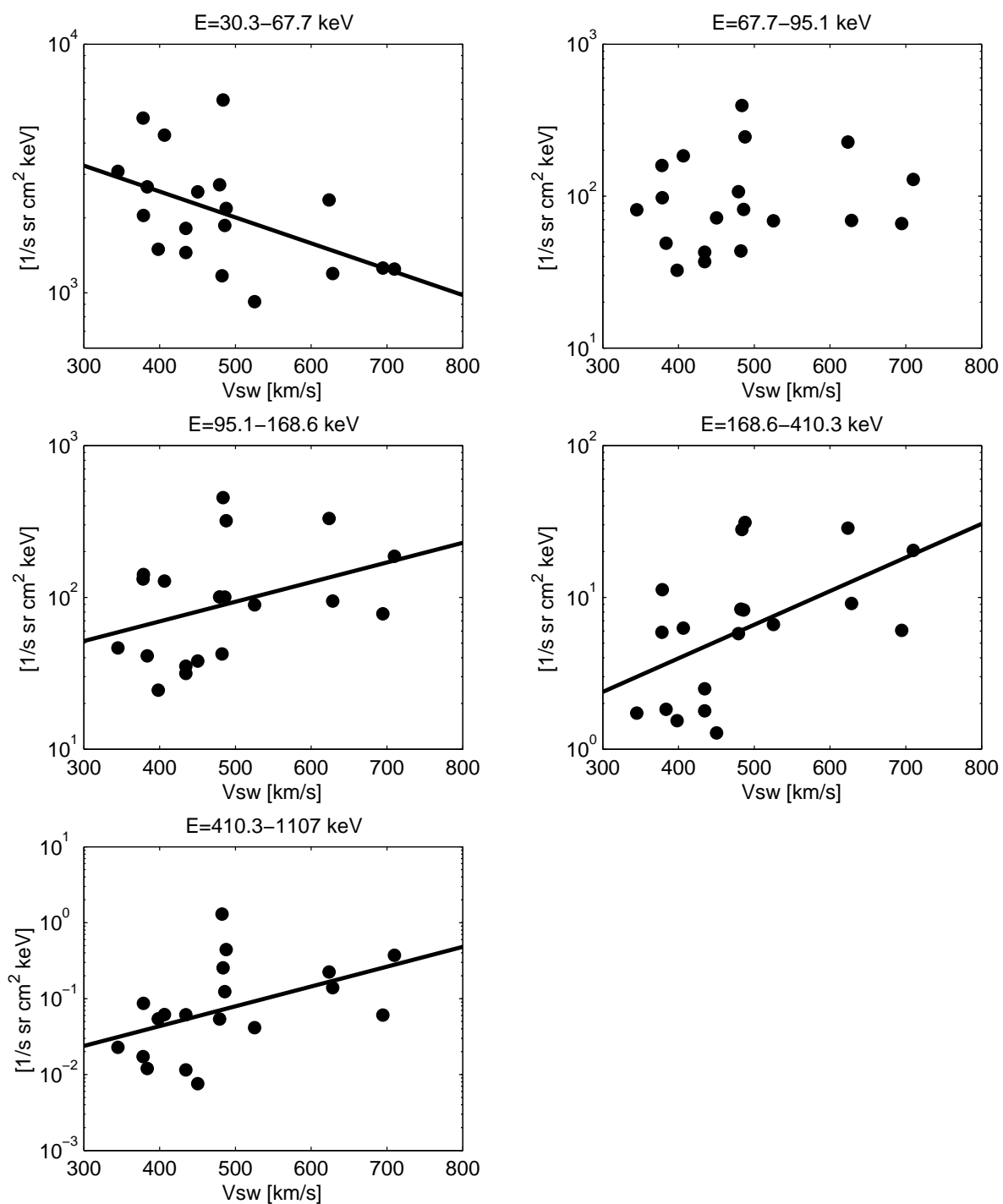

Fig. 8. Differential proton fluxes (five lowest energy channels) in the exterior cusp as a function of solar wind speed.

Table 4. Spearman correlation coefficients between differential proton fluxes in the exterior cusp and substorm activity (AE index) in different energy ranges.

\begin{tabular}{llll}
\hline $\begin{array}{l}\text { Energy range } \\
{[\mathrm{keV}]}\end{array}$ & $\begin{array}{l}\text { Spearman } \\
\text { correlation }\end{array}$ & P-value & $\begin{array}{l}\text { Slope of } \\
\text { fit }\end{array}$ \\
\hline $30.3-67.7$ & -0.03 & 0.45 & 0.0003 \\
$67.7-95.1$ & 0.51 & 0.01 & 0.0024 \\
$95.1-168.6$ & 0.68 & 0.0007 & 0.0036 \\
$168.6-410.3$ & 0.64 & 0.002 & 0.0043 \\
$410.3-1107$ & 0.45 & 0.03 & 0.0039 \\
\hline
\end{tabular}

when the solar wind speed is higher. Contrary to the protons the electrons show the same behaviour on all energy channels. The Spearman correlation between the total electron flux and the solar wind speed is 0.46 with a $\mathrm{P}$-value of 0.03 .
Figure 9 displays the differential proton fluxes with respect to the AE index. The lower energy fluxes do not clearly show any dependence on the AE index. However, at higher energy channels the fluxes clearly correlate with substorm activity. It is interesting to note that the dependence on substorm activity appears at higher energies while the dependence on the magnitude of IMF $B_{y}$ is more evident at lower energies. The Spearman correlation coefficients and their significance for the five lowest proton energy channels are displayed in Table 4 .

Figure 10 shows the total energetic electron fluxes in the exterior cusp as a function of AE index. One can see a rather clear dependence in the plot. The Spearman correlation between the electron fluxes and the AE index is $0.35(\mathrm{P}=0.07)$. The same dependence on the substorm activity was seen in all electron energy channels. 

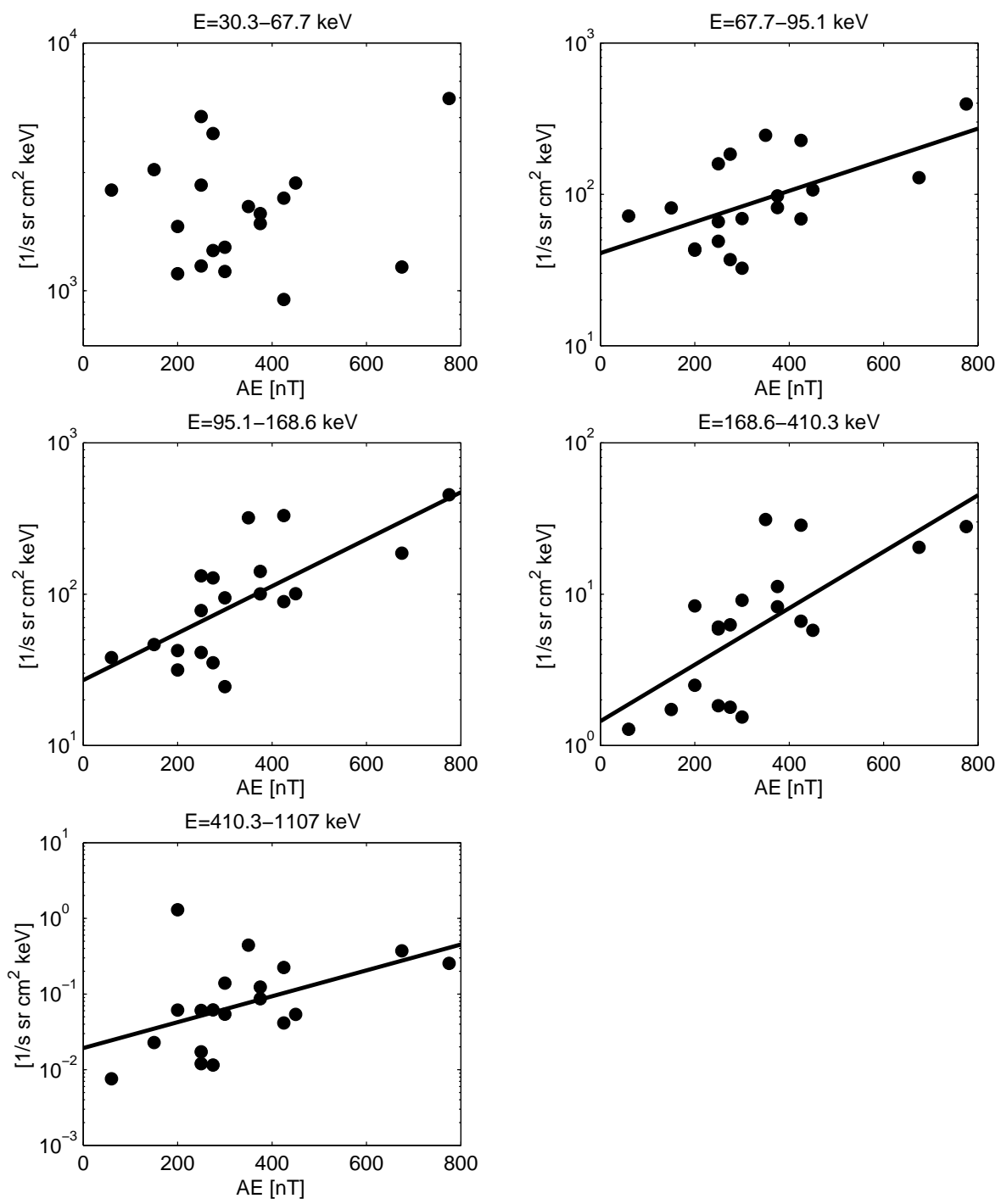

Fig. 9. Differential proton fluxes observed in the exterior cusp at the five lowest energy channels as a function of substorm activity (AE index).

\subsection{HLPS}

Let us now examine how the energetic particle fluxes in the high-latitude dayside plasma sheet depend on the solar wind and IMF conditions. Figure 11 shows the relation between the energetic proton and electron fluxes and the solar wind dynamic pressure. (Note that there are only 16 data points in these plots since there was no solar wind density data available during one HLPS pass.) Proton fluxes increase with dynamic pressure with a correlation coefficient of 0.52 $(\mathrm{P}=0.02)$. However, there is no such trend for electrons. (The Spearman correlation for electron fluxes is $-0.25(\mathrm{P}=0.17)$ and shows that the correlation is weak and statistically not significant.)

Figure 12 displays the total proton and electron fluxes in HLPS as a function of solar wind speed. The proton fluxes do not show any dependence on solar wind speed and this is true for all proton energy channels. Contrary to

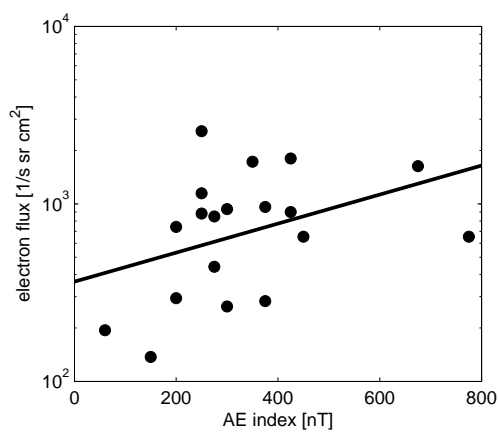

Fig. 10. Total energetic electron fluxes in the exterior cusp as a function of AE index.

protons, electrons depict quite a clear dependence on solar wind speed. The Spearman correlation for electron fluxes is $0.59(\mathrm{P}=0.006)$. The same relation is seen on every energy channel for electrons. 

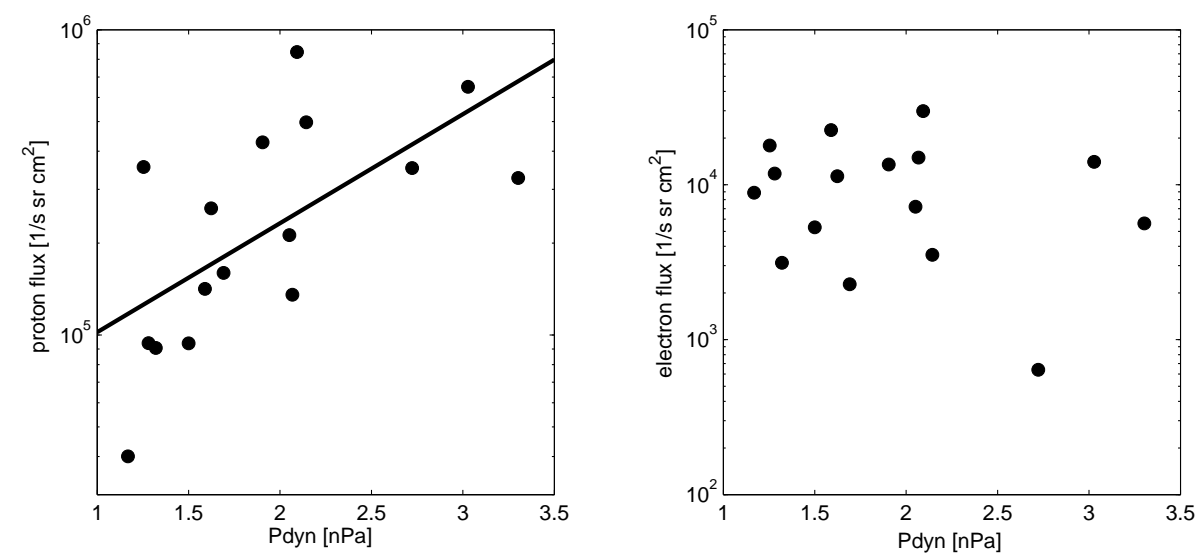

Fig. 11. Energetic particle fluxes in the HLPS as a function of solar wind dynamic pressure. Left: protons, right: electrons.
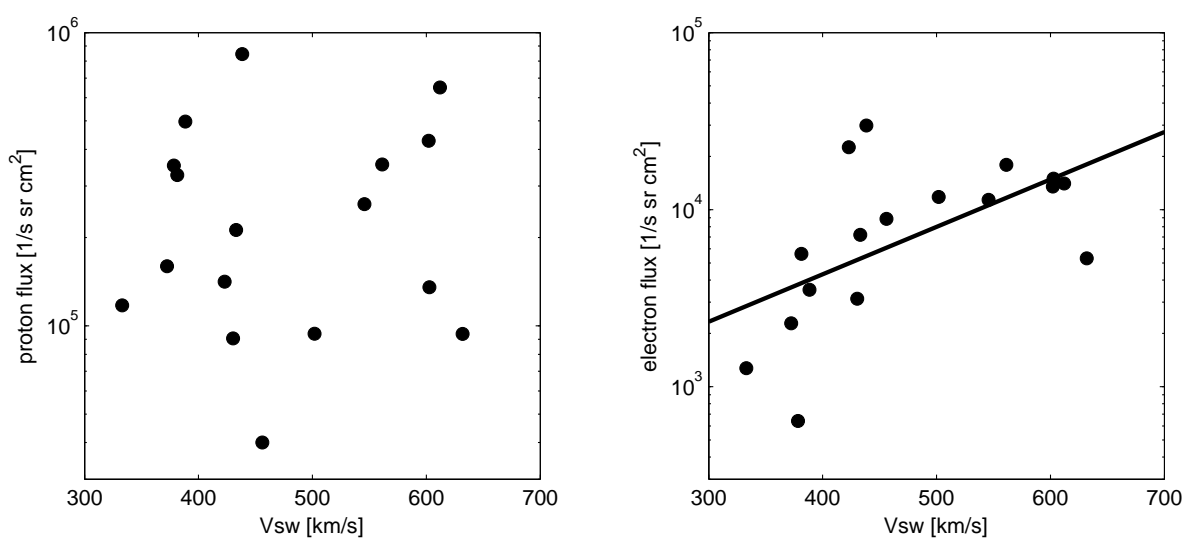

Fig. 12. Energetic particle fluxes in the HLPS as a function of solar wind speed. Left: protons, right: electrons.

We have also studied the relation between the IMF components and the energetic particle fluxes in the HLPS but did not find any statistically significant correlations.

Let us now study how the energetic particle fluxes in the HLPS depend on geomagnetic activity. Figure 13 displays the total proton and electron fluxes in the HLPS as a function of the 3-h averaged AE index. For protons there is a very clear dependence on the substorm activity and this same relation is seen on every energy channel. The correlation between the proton flux and the AE index is $0.80(\mathrm{P}=0.00005)$. Perhaps surprisingly, there is no clear and statistically significant relation between the electron fluxes and the AE index on any energy channel.

We have also studied the possible dependence of the energetic particle fluxes in the HLPS on the geomagnetic $K_{p}$ index (see Fig. 14), and found a very similar relation in the proton fluxes as for the AE index. There is a highly significant correlation between proton fluxes and the $K_{p}$ index, with a correlation coefficient $0.68(\mathrm{P}=0.002)$. Interestingly, we also found a weakly significant relation between the $K_{P}$ index and the electron fluxes. When we leave out the outlier, with an unusually low electron flux, the correlation coefficient is $0.52(\mathrm{P}=0.02)$. Using all the data points, the corre- lation is $0.35(\mathrm{P}=0.08)$. We have summarized the different correlations and their significances in Table 5.

\section{Comparison between HLPS and exterior cusp}

A very efficient method to investigate the possible acceleration mechanisms of particle populations is to look at their spectral characteristics. For this purpose we have fitted a power law spectrum to the data and calculated the average spectral indices on the basis of the five lowest energy channels for electrons and protons in the exterior cusp and HLPS. For protons the average spectral index was $3.5 \pm 0.6$ in both regions and for electrons the average spectral index was $3.4 \pm 0.5$ in the HLPS and 3.6 \pm 0.4 in the exterior cusp.

Let us next directly compare the fluxes between the HLPS and exterior cusp. This comparison makes sense only on those orbits where the satellite detects both regions. Rather surprisingly, orbits showing clear signals of both regions were rare. We found only three such orbits, one of which where the cusp was detected twice, thus giving four pairs to compare. Above we showed that there were considerable differences in the behaviour of protons at different energies. Therefore, we have compared the different proton 

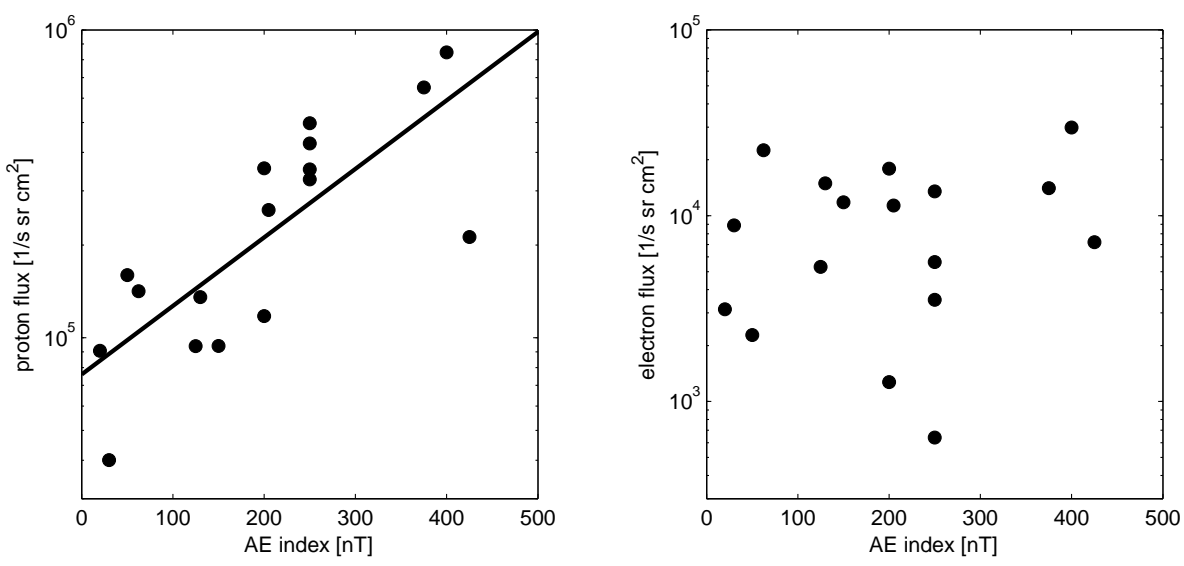

Fig. 13. Energetic particle fluxes in the HLPS as a function of 3-h averaged AE index. Left: protons, right: electrons.
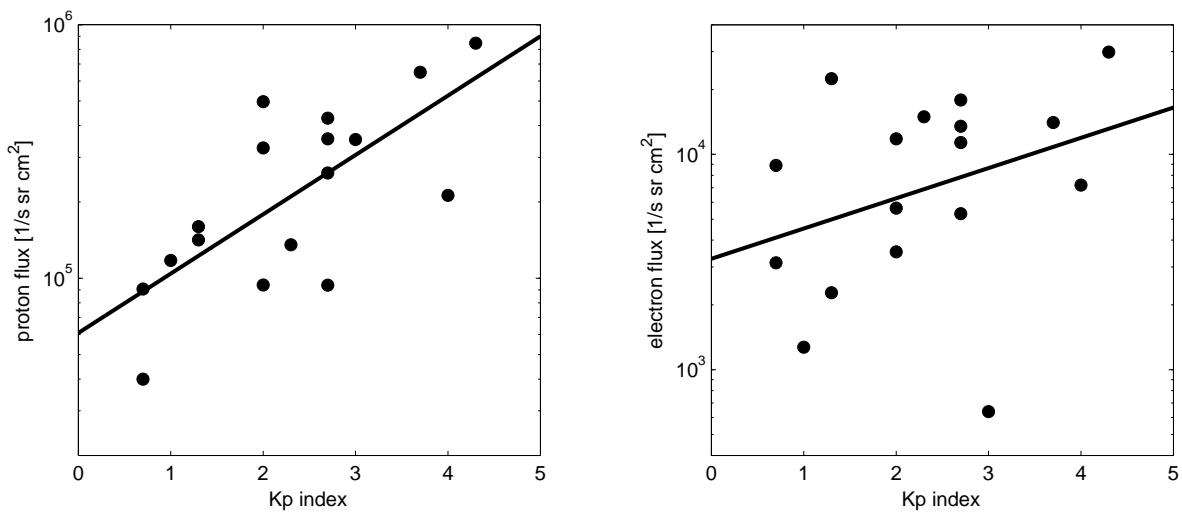

Fig. 14. Energetic particle fluxes in the HLPS as a function of $K_{P}$ index. Left: protons, right: electrons.

energy channels separately instead of using energy integrated fluxes. We calculated the (Pearson) correlation coefficients between the logarithmic proton fluxes in the exterior cusp and HLPS. In the lowest energy channel the correlation was $-0.48(\mathrm{P}=0.53)$, thus insignificant. However, when the higher energy channels were considered separately the correlation was in the range $0.53-0.77$ (average $\mathrm{P} \approx 0.4$ ), which indicates a fair correlation, despite the low number of events. The correlation between the energy integrated electron fluxes in the two regions was $0.90(\mathrm{P}=0.11)$. Thus, there is a strong and significant correlation between the electron fluxes in the exterior cusp and HLPS. This is further supported by the fact that there is a fair correlation (correlation coefficient $=0.93$ 0.97 and $\mathrm{P} \approx 0.04$ ) even for separate electron energy channels.

\section{Discussion}

The present observations clearly show that there is a quasipermanent energetic particle population in the tens to hundreds of $\mathrm{keV}$ energy range, in the exterior cusp and in the high-latitude dayside plasma sheet. Figure 2 shows that the average proton (electron) fluxes are roughly three (ten) times larger in the HLPS region than in the exterior cusp. This is also true for the (nearly) simultaneously observed fluxes on those Cluster orbits when both regions were visited during a short time interval. We note that the proton fluxes in both regions are over two orders of magnitude higher than the electron fluxes. This cannot be due to the slightly different energy intervals of the RAPID instrument, since the threshold energy is even smaller for electrons than for protons.

Let us first discuss the energetic particle fluxes in the closed field-line region of the high-latitude dayside plasma sheet. In this region the proton fluxes are strongly correlated with $\mathrm{AE}$ and $K_{P}$ indices, independent of proton energy. This relation strongly suggests that the energetic protons in the HLPS have been accelerated in the substorm processes, in the near-Earth magnetotail. It has been shown (e.g. Delcourt and Sauvaud, 1999) that energetic particles can drift from the near-Earth tail to the dayside magnetosphere and subsequently rise to the HLPS region. The particles can reach the HLPS because near the dayside magnetopause the magnetic field minimum along a field line is located in the HLPS region, whence the magnetic gradient force at the subsolar region points to high latitudes. Moreover, numerical simulations have shown that in a rather stable magnetic field 
Table 5. Summary of different correlation coefficients and their significances.

\begin{tabular}{lll}
\hline Parameters & $\begin{array}{l}\text { Spearman } \\
\text { correlation }\end{array}$ & P-value \\
\hline Exterior cusp & & \\
Proton flux vs. IMF $\left|B_{y}\right|$ & 0.67 & 0.0008 \\
Electron flux vs. IMF $B_{z}$ & 0.45 & 0.03 \\
Diff. Proton flux vs. $V_{s} w$ & & \\
(4th energy channel) & 0.59 & 0.004 \\
Electron flux vs. $V_{s} w$ & 0.46 & 0.03 \\
Electron flux vs. AE index & 0.35 & 0.07 \\
HLPS & & \\
Proton flux vs. solar wind pressure & 0.52 & 0.02 \\
Electron flux vs. solar wind pressure & -0.25 & 0.17 \\
Electron flux vs. $V_{s w}$ & 0.59 & 0.006 \\
Proton flux vs. AE index & 0.80 & 0.00005 \\
Proton flux vs. $K_{p}$ index & 0.68 & 0.002 \\
Electron flux vs. $K_{p}$ index & 0.52 & 0.02 \\
Correlations between HLPS and & & \\
exterior cusp fluxes & & \\
Diff. Proton fluxes $(E<68 \mathrm{keV})$ & -0.48 & 0.53 \\
Diff. Proton fluxes $(E>68 \mathrm{keV})$ & 0.69 & 0.3 \\
Electron fluxes & 0.90 & 0.11 \\
\hline
\end{tabular}

the energetic particles can drift around the cusp funnel in the high-latitude dayside plasma sheet and be quasi-trapped (Sheldon et al., 1998). On this basis it is rather easy to understand the origin of fairly large and persistent fluxes of energetic particles in the HLPS.

Unlike the protons the electrons in the HLPS did not show any clear correlation with substorm activity. They did depict a weak dependence on the $K_{P}$ index which describes global magnetospheric disturbances at mid-latitudes, i.e. closer to the Earth than the processes described by the AE index. However, the strongest correlation was found with the solar wind speed, which seems to be the dominant factor for the energetic electron fluxes in the HLPS. It is well known that the enhancements of energetic electrons in the outer radiation belt are correlated with solar wind speed (see, e.g. Paulikas and Blake, 1979 and Li et al., 1997). The similar relation found between electron fluxes in the HLPS and solar wind speed suggests that these electrons are related to the energetic electrons in the outer radiation belt. Electrons can access the HLPS in the same manner as protons.

The protons in the HLPS showed significant correlation with solar wind dynamic pressure at all energies. This may be due to the effect of solar wind pressure on the structure of the dayside magnetosphere. When pressure increases the magnetosphere is compressed and the dayside magnetopause shifts inward. Since the effects of pressure are strongest in the subsolar region (where the flow is perpendicular to the magnetopause) the magnetopause shifts inward more than at higher latitudes. Thus, the ratio of subsolar and HLPS magnetic field strengths increases as pressure increases. This in turn creates a more efficient magnetic trap in the HLPS region and increases the energetic proton fluxes.
Let us now turn to discuss particles in the exterior cusp. The observations showed that the behaviour of proton fluxes in the exterior cusp was strongly energy dependent, unlike in the HLPS. At lower energies (below about $100 \mathrm{keV}$ ) the proton fluxes were strongly correlated with the IMF $\left|B_{y}\right|$ component while at higher energies the fluxes were found to depend mainly on the substorm (or geomagnetic) activity as measured by the $\mathrm{AE}\left(K_{P}\right)$ index. The dependence on substorm activity at higher energies suggests that these protons are connected to substorm processes in the tail. Also, given the good correlation between the fluxes of higher-energy protons in the two regions, it seems that the higher-energy protons can diffuse from the adjacent HLPS to the exterior cusp. The lack of correlation between the lower-energy fluxes and the $\mathrm{AE}$ index, on one hand, and the lower-energy fluxes in the two regions, on the other hand, can only mean that the lower-energy protons do not significantly diffuse directly to the cusp. The correlation between the IMF $\left|B_{y}\right|$ and the lower-energy proton fluxes in the exterior cusp suggests that the IMF has some part to play in either accelerating or transporting these particles to the exterior cusp. One possible explanation might be reconnection in the dayside high-latitude magnetopause. When the IMF $B_{y}$ component increases relative to the other components, the site of antiparallel reconnection moves from the subsolar region (dominant $B_{z}<0$ ) or lobe (dominant $B_{z}>0$ ) to the dawn or dusk edge of the HLPS region, where the field lines bend to form the cusp funnel and are thus locally nearly horizontal. If reconnection happens in this region, it obviously could release plasma and energetic particles from the HLPS to the newly-opened field lines connected to the cusp.

Another possibility is that the lower-energy protons would originate somewhere else than in the HLPS and be accelerated by some other mechanism than substorm acceleration. In this case the efficiency of acceleration and/or transport of protons should depend on the IMF $\left|B_{y}\right|$. One possible IMF dependent source could be bow shock acceleration. While this mechanism may produce some of the observed lowerenergy protons it is unlikely to be the major source. During high IMF $\left|B_{y}\right|$ the quasi-parallel region of the bow shock (where the acceleration presumably would be most efficient) would be far in the dawn and dusk flanks of the magnetosphere. If the particles were accelerated there, their transport to the exterior cusp would become more difficult with increasing $\left|B_{y}\right|$.

Energetic electrons in the exterior cusp were found to behave differently from the protons. In contrast to protons, electrons in all the studied energy channels behaved quite similarly. While the low energy proton fluxes showed correlation with the IMF $\left|B_{y}\right|$ component the electron fluxes correlated weakly with the $B_{z}$ component, with the fluxes being higher for positive $B_{z}$. This can also be explained in terms of reconnection. When $B_{z}$ is positive the antiparallel reconnection site is poleward of the cusp in the high-latitude boundary layer or the plasma mantle. In this case the topology of cusp field lines is more closed and stable than during southward IMF, since the reconnected field lines try to move sunward 
against the tailward flow of the solar wind. This sort of closed cusp probably confines the fast electrons better than the more open cusp geometry during southward IMF.

The fact that the energetic electron fluxes in the exterior cusp correlated with the solar wind speed suggests that these electrons may be accelerated by the same mechanism as the outer radiation belt electrons. Interestingly, the electron fluxes in the cusp also correlated with the AE index. This is in contrast to the electron fluxes in the HLPS which showed hardly any correlation with AE. In order to further study this puzzling relation we performed a partial correlation analysis of the electron fluxes and the various variables (like $\mathrm{AE}$ and $\left.V_{s w}\right)$. We found out that on the studied Cluster cusp passes the $\mathrm{AE}$ and solar wind speed correlated significantly with a correlation coefficient of $0.47(\mathrm{P}=0.02)$. Furthermore, partial correlation analysis revealed that the seeming AE dependence of the electron fluxes in the cusp was actually dependence on solar wind speed. This fact supports the idea that the cusp electrons are from the same source as the electrons in the HLPS, i.e. outer radiation belt.

In addition to the evidence presented and discussed above, there are also other facts supporting the idea that the energetic particles in the exterior cusp originate mainly in the HLPS. The similar spectral indices for protons and electrons in the exterior cusp and the HLPS suggest a similar acceleration mechanism for both particle species and both regions. The evidence is further increased by the correlation between the roughly simultaneous particle fluxes in the two regions on those Cluster orbits where both regions were visited.

Let us now discuss in more detail the nature of the mechanism that transports the particles from the HLPS to the exterior cusp. The strong dependence of the lower-energy proton fluxes on IMF $\left|B_{y}\right|$ suggests that the magnetic reconnection in the HLPS region releases these protons to the cusp. It would be natural to expect that reconnection would also release higher-energy protons and energetic electrons. Yet the IMF $\left|B_{y}\right|$ dependence is not seen in the higher-energy protons or in the electrons. Apparently, the higher-energy protons and electrons in the exterior cusp have reached this location by a mechanism which is independent of reconnection. This mechanism cannot be mass, energy or gyroradius dependent, since it would also affect the lower-energy protons. The only possibility is that the transport mechanism has to depend on the velocity of the particle. If this mechanism only affects protons above $100 \mathrm{keV}$, the affected electrons must have an energy of about $60 \mathrm{eV}$. Thus, all the electrons measured by RAPID would be affected by the mechanism and thus behave similarly as the higher energy protons. The most natural mechanism that comes into question is diffusion of particles caused, for example by scattering from small disturbances on magnetic field lines. The higher velocity of a particle increases the probability to meet such disturbances.

Finally, let us discuss the two other models that have been suggested to explain the origin of energetic ions in the exterior cusp. One is the bow shock acceleration model by Chang et al. (1998) and Trattner et al. (2001), who found that the cusp energetic ion fluxes increased for positive IMF $B_{z}$ and small cone angle (essentially, large $B_{x}$ ). Both of these results are in contradiction with our observations. Furthermore, the bow shock acceleration cannot naturally explain the energetic electron fluxes observed in the cusp. The other model suggested for the energetic ions in the cusp is based on the local acceleration of ions. Chen et al. (1998) observed a correlation between the He ion fluxes and ULF turbulence, suggesting that such a turbulence could accelerate the ions. Despite the observed correlation, it gives no direct evidence in favour of strong local acceleration, since turbulence may be a result rather than cause of increased ion fluxes. Moreover, the energy threshold of the observed He ions was only $1 \mathrm{keV} / \mathrm{e}$, thus much below the energy interval studied here. Another argument suggested in favour of both the bow shock and the acceleration model is the relatively large fraction of solar wind ions in the cusp (Chen et al., 1998). However, as noted above, the energy threshold is much lower than that used here, and is fairly close to the thermal energy of magnetosheath ions. Therefore, the observed ion ratios do not yield strong evidence in favour of either model. Finally, we note that neither of these two models can explain both energetic ion and electron fluxes in the cusp within one single mechanism.

\section{Conclusions}

In this paper we have studied the fluxes of energetic protons $(40-4000 \mathrm{keV})$ and electrons $(30-400 \mathrm{keV})$ in the exterior cusp and in the adjacent high-latitude dayside plasma sheet with the Cluster/RAPID instrument. Using two sample orbits we demonstrated that the Cluster observations at high latitudes can be dramatically different when the satellite orbit traverses different plasma regions for different external conditions. We have made a statistical study of energetic particles in the exterior cusp and the HLPS by analysing all outbound Cluster dayside passes in February and March, 2002 and 2003.

First of all, our results verify that there are significant fluxes of energetic particles both in the exterior cusp and HLPS. The average particle fluxes in the HLPS are roughly three (protons) or ten (electrons) times larger than in the exterior cusp. This is also true on those Cluster orbits where both regions are visited within a short time interval. Moreover, the total electron fluxes, as well as proton fluxes above some $100 \mathrm{keV}$, in these two regions correlate with each other. This is true even for fluxes in every energy channel when considered separately. The spectral indices of electron and proton fluxes are found to be the same in the two regions.

We have also examined the possible dependence of particle fluxes at different energies on the external (solar wind and IMF) and internal (geomagnetic) conditions. The energetic proton fluxes (but not electron fluxes) in the cusp were found to behave differently at low and high energies. At low energies $(<70 \mathrm{keV})$, the fluxes increased strongly with the magnitude of IMF $B_{y}$. Instead, at higher energies the proton fluxes in the cusp depended on substorm/geomagnetic 
activity. In HLPS proton fluxes, irrespective of energy, depended strongly on the $K_{p}$ and $\mathrm{AE}$ indices. The electron fluxes in HLPS depended both on the $K_{p}$ index and the solar wind speed. In the cusp the electron fluxes mainly depended on the solar wind speed, and were higher for northward than southward IMF. The HLPS and cusp electrons depend, in the same way as energetic electrons in the outer radiation belt, on solar wind speed, indicating that the HLPS/cusp electrons and outer radiation elecrons are related.

These results give strong evidence in favour of the idea that the high-latitude dayside plasma sheet is the main source of energetic particles in the exterior cusp. Energetic particles can reach HLPS from the near-Earth tail. This is supported by the fact that particle fluxes in the HLPS were clearly connected with the acceleration processes related to substorms in the near-Earth magnetotail. The closed field lines of HLPS act as storage for these particles. Electrons and high-energy protons enter the exterior cusp from the HLPS mainly via direct diffusion. On the other hand, magnetic reconnection in the high-latitude magnetopause near the HLPS acts to control the number of low energy protons released into the exterior cusp. Thus, ultimately, energetic protons in the exterior cusp have been accelerated in the magnetospheric tail by processes related to the substorm cycle and electrons probably in stochastic processes accelerating the outer radiation belt electrons. Note that this explanation, in contrast to other suggested theories, works both for the energetic protons and electrons in the exterior cusp.

Acknowledgements. This work was done during the ANTARES space research program and funded by the Academy of Finland. We wish to thank the Cluster RAPID, FGM, CIS and EFW teams and the instrument PIs (P. Daly, A. Balogh, H. Rème and M. Andrè) for data.

Topical Editor T. Pulkkinen thanks R. Friedel and another referee for their help in evaluating this paper.

\section{References}

Balogh, A., Dunlop, M., Cowley, S., et al.: The Cluster Magnetic Field Investigation, Space Science Reviews, 79, 65-91, 1997.

Chang, S.-W., Scudder, J., Fuselier, S., et al.: Cusp energetic ions: A bow shock source, Geophys. Res. Lett., 25, 3729-3732, 1998.

Chen, J., Fritz, T. A., Sheldon, R. B., et al.: Cusp energetic particle events: Implications for a major acceleration region of the magnetosphere, J. Geophys. Res., 103, 69-78, 1998.

Chen, J., Fritz, T. A., Sheldon, R., Pickett, J., and Russell, C.: The discovery of a new acceleration and possible trapping region of the magnetosphere, Adv. Space Res., 27, 1417-1422, 2001.
Delcourt, D. and Sauvaud, J.-A.: Populating of Cusp and Boundary Layers by energetic (hundreds of $\mathrm{keV}$ ) equatorial particles, J. Geophys. Res., 104, 22, 635-22, 648, 1999.

Fritz, T. A.: The Cusp as a source of magnetospheric energetic particles, currents, and electric fields: A New Paradigm, Space Science Reviews, 95, 469-488, 2001.

Fritz, T. A., Chen, J., Sheldon, R., Spence, H., Fennell, J., Livi, S., Russell, C., and Pickett, J.: Cusp Energetic Particle Events Measured by POLAR Spacecraft, Phys. Chem. Earth, 24, 135140, 1999.

Fritz, T. A., Chen, J., and Siscoe, G.: Energetic ions, large diamagnetic cavities and Chapman-Ferraro cusp, J. Geophys. Res., 108, 1028, doi:10.1029/2002JA009476, 2003.

Gustafsson, G., Boström, R., Holback, B., et al.: The Electric Field and Wave experiment for the Cluster mission, Space Science Reviews, 79, 137-156, 1997.

Kremser, G., Woch, J., Mursula, K., Tanskanen, P., Wilken, B., and Lundin, R.: Origin of energetic ions in the polar cusp inferred from ion composition measurements by the Viking satellite, Ann. Geophys., 13, 595-607, 1995,

SRef-ID: 1432-0576/ag/1995-13-595.

Li, X., Baker, D., Temerin, M., et al.: Are energetic electrons in the solar wind the source of the outer radiation belt?, Geophys. Res. Lett., 24, 923-926, 1997.

Mursula, K., Kerttula, R., Asikainen, T., et al.: CLUSTER/RAPID energetic electron observations at the dayside magnetospheric boundary, Adv. Space Res., in press, 2005.

Paulikas, G. and Blake, J.: Effects of the solar wind on magnetospheric dynamics: Energetic electrons at the synchronous orbit, in Quantitative Modeling of Magnetospheric Processes, Vol. 21 of Geophysical Monograph, American Geophysical Union, 1979.

Rème, H., Bosqued, J. M., Sauvaud, J. A., et al.: The Cluster Ion Spectrometry (CIS) Experiment, Space Sci. Rev., 79, 303-350, 1997.

Roederer, J.: Dynamics of geomagnetically trapped radiation, Springer-Verlag Berlin Heidelberg New York, 1970.

Rosenbauer, H., Grünwaldt, H., Montgomery, M., Paschmann, G., and Sckopke, N.: Heos 2 Plasma Observations in the Distant Polar Magnetosphere: The Plasma Mantle, J. Geophys. Res., 80, 2723-2737, 1975.

Sheldon, R., Spence, H., Sullivan, J., Fritz, T. A., and Chen, J.: The discovery of trapped energetic electrons in the outer cusp, Geophys. Res. Lett., 25, 1825-1828, 1998.

Stone, E., Frandsen, A., Mewaldt, R., Christian, E., Margolies, D., Ormes, J., and Snow, F.: The Advanced Composition Explorer, Space Science Reviews, 86, 1-22, 1998.

Trattner, K., Fuselier, S., Peterson, W., Chang, S.-W., Friedel, R., and Aellig, M.: Origins of energetic ions in the cusp, J. Geophys. Res., 106, 5967-5976, 2001.

Wilken, B., Axford, W., Daglis, I., et al.: RAPID: The Imaging Energetic Particle Spectrometer on Cluster, Space Science Reviews, 79, 399-473, 1997. 